\title{
Endogenous $A \beta$ induces osteoporosis through an mTOR- dependent inhibition of autophagy in bone marrow mesenchymal stem cells (BMSCs)
}

\author{
Ye $\operatorname{Lin}^{1} \wedge^{\#}$, Tianyu Chen ${ }^{2 \#}$, Junjian Chen ${ }^{1 \#}$, Yingying Fang ${ }^{3}$, Canjun Zeng ${ }^{1}$ \\ ${ }^{1}$ Department of Foot and Ankle Surgery, Center for Orthopedic Surgery, the Third Affiliated Hospital of Southern Medical University, Guangzhou, \\ China; ${ }^{2}$ Department of Spinal Surgery, Center for Orthopedic Surgery, the Third Affiliated Hospital of Southern Medical University, Guangzhou, \\ China; ${ }^{3}$ Southern Medical University, Guangzhou, China \\ Contributions: (I) Conception and design: Y Lin, C Zeng; (II) Administrative support: Y Lin, C Zeng; (III) Provision of study materials or patients: \\ Y Lin, J Chen; (IV) Collection and assembly of data: Y Lin, T Chen; (V) Data analysis and interpretation: Y Lin, C Zeng, Y Fang; (VI) Manuscript \\ writing: all authors; (VII) Final approval of manuscript: all authors. \\ \#These authors contributed equally to this work and should be considered as co-first authors. \\ Correspondence to: Dr. Canjun Zeng. Department of Foot and Ankle Surgery, Center for Orthopedic Surgery, the Third Affiliated Hospital of \\ Southern Medical University, No 184, Zhongshan Road, Guangzhou, China. Email: zengcanjun@163.com.
}

Background: It has previously been suggested that Alzheimer's disease (AD) and osteoporosis (OP) were related. However, the connection between these 2 disorders is poorly understood. This study aimed to investigate the relationship between amyloid $\beta$ peptide $(\mathrm{A} \beta)$ and the osteoporotic deficit observed in $\mathrm{AD}$ patients.

Methods: We used the APP/PS1 $\triangle \mathrm{E} 9$ transgenic mouse model of AD for in vivo study and extracted bone marrow mesenchymal stem cells (BMSCs) for in vitro studies. For in vivo experiments, mice femurs were put through a $\mu$-computer tomography ( $\mu$-CT) scanning and after which, sliced for hematoxylin/eosin (HE), Masson and Goldner staining for detection of bone changes. For in vitro experiments, BMSCs were placed in an osteogenic inducing medium with or without rapamycin. After induction, alkaline phosphatase (ALP) staining, alizarin red staining, quantitative real-time PCR (qPCR) and western-blot were used to identify osteogenic differentiation, calcium deposition and protein expression differences respectively.

Results: We observed that pathological changes characteristic of $\mathrm{AD}$ and $\mathrm{OP}$ occurred in vivo in APP/ PS1 $\triangle \mathrm{E} 9$ mice. In BMSCs producing endogenous $\mathrm{A} \beta$, mammalian target of rapamycin (mTOR) activation and subsequent inhibition of autophagy suppressed bone formation. Further, the addition of the mTOR inhibitor rapamycin into the inducing medium reversed the inhibition of osteogenesis.

Conclusions: Our results suggested that endogenous $\mathrm{A} \beta$ might have induced osteoporosis through an mTOR-dependent inhibition of autophagy in BMSCs, which may explain the OP changes observed in AD patients.

Keywords: Amyloid $\beta$ (Aß); osteoporosis; Alzheimer's disease (AD); bone marrow mesenchymal stem cells (BMSCs); mTOR

Submitted Aug 10, 2021. Accepted for publication Dec 22, 2021.

doi: $10.21037 / \mathrm{atm}-21-6427$

View this article at: https://dx.doi.org/10.21037/atm-21-6427

^ ORCID: 0000-0003-1189-0822. 


\section{Introduction}

Alzheimer's disease (AD) and osteoporotic deficit are multifactorial progressive degenerative disorders that have been found to be epidemiologically connected $(1,2)$. Osteoporotic defects and hip fracture are common complications observed in $\mathrm{AD}$ patients, and the risk of developing osteoporotic hip fractures is higher for $\mathrm{AD}$ patients, even after accounting for the increased frequency of falls (2-5). Further, compared to the general aging population, lower bone mineral density (BMD) has been reported in the early stages of $\mathrm{AD}$ before neural degeneration and mobility impairment are detected, common occurrence of osteoporotic changes were reported in early or late stage of $\mathrm{AD}$, but little of $\mathrm{AD}$ changes were observed even in elder osteoporosis patients, taken together, osteoporotic changes were considered to be early common pathological symptoms caused by $\mathrm{AD}$ pathogenic factor in some AD patients. Still, mechanism underlying osteoporotic defects in $\mathrm{AD}$ patents are unclear (4-7). Oxidative stress, inflammation, autophagy, and apoptosis are involved in these age-related diseases (8). However, the mechanisms underlying the association between the pathogenic factor of $\mathrm{AD}$ and osteoporotic deficit remain poorly understood (1).

Deposition of amyloid $\beta$ peptide $(A \beta)$ in the brain in the form of oligomeric $A \beta$ and $A \beta$ plaques is one of the key pathological hallmarks of $\mathrm{AD}(9,10) . \mathrm{A} \beta$ is a small proteolytic fragment derived from amyloid precursor protein (APP), an integral membrane cell surface receptor protein encoded by a gene on human chromosome 21 (11). As $A \beta$ is believed to play a crucial role in $\mathrm{AD}$ pathogenesis, it is possible that it is a key pathogenic factor causing the associated osteoporotic deficit. Excessive accumulation of $A \beta$ in tissues may jeopardize biological functions, leading to $\mathrm{AD}$ and osteoporosis (OP). An osteoporotic phenotype of low BMD has been observed in transgenic mice overexpressing $A \beta$ and has been used in $\mathrm{AD}$ studies $(3,12-14)$. However, there is little evidence available to confirm a shared mechanism for $\mathrm{AD}$ and $\mathrm{OP}$ in humans.

APP/PS1 mice are double transgenic mice with overexpression of mouse/human amyloid precursor protein (APPswe) and mutant human presenilin 1 (PS1-dE9), both mutations are related to Alzheimer's disease. These transgenic mice were reported to develop $A \beta$ in brain by 6 to 7 months of age, and the accumulation of $A \beta$ is more in the form of molecules than in granulation $(15,16)$ These mice were generally used in studying neurological disorders of the brain, specifically Alzheimer's disease and amyloid plaque formation (15-17).

Bone is a hard, highly mineralized tissue which undergoes lifelong remodeling to sustain its stiffness and intensity and to maintain its biological function. The major processes involved in bone remodeling are bone grafting by osteoblasts and resorption by osteoclasts (18). The malfunction of either type of factor might induce the hyperactivity of osteoclasts or weakening of osteoblasts, leading to an imbalance between bone resorption and formation, causing OP $(18,19)$.

The relationship between $\mathrm{A} \beta$ and bone metabolism has not been clearly determined. A previous study indicated that $A \beta$ enhanced osteoclast activation by receptor activator of nuclear factor kappa-B ligand (RANKL) through I $\kappa \mathrm{B}-\alpha$ degradation, extracellular signal-regulated kinase (ERK) phosphorylation, and calcium oscillation. Therefore, $A \beta$ may mediate the decrease in BMD and OP (20). Another study showed that $\mathrm{A} \beta$ treatment simultaneously promoted osteogenic differentiation of osteoblasts via $\mathrm{Wnt} / \beta$-catenin signaling and inhibited osteoclast differentiation through the osteoprotegerin (OPG)/RANKL/RANK system (21). However, these studies used exogenous $A \beta(20,21)$, which is very different from the pathological process of endogenous $\mathrm{A} \beta$ generated intracellularly and ultimately deposited in extracellular matrix. Moreover, the bone phenotypes of exogenous $A \beta$ experiments were completely unlike the observed bone loss symptoms in $\mathrm{AD}$ patients and the low $\mathrm{BMD}$ present in transgenic $\mathrm{AD}$ mice $(3,12)$.

Mammalian target of rapamycin (mTOR), receives various stimulation from intracellular and extracellular changes, the activated mTOR promotes cell anabolism to produce kinds of molecules which are in charge of ribosome genesis, gene expression, and protein translation. Activation of mTOR can lead to blockage of catabolism such as lysosome genesis and autophagy, in which, mTOR plays a crucial switch role (22). The mTOR integrates with range of cytokines and pathways up or downstream to modulate metabolic pathways for cell proliferation, growth, and metabolism $(22,23)$. mTOR signaling has been proven to be crucial in regulating synapse formation and plasticity $(24,25)$. Moreover, inhibition of mTOR signaling improves spatial learning, memory, and AD-like pathology in APP/PS1 $\triangle \mathrm{E} 9$ mice (hereafter referred to as APP/PS1 mice), autophagy might play a regulatory role in this process (26-29). Previous studies have found that in osteogenesis, osteoblast proliferation was accompanied by increased mTOR phosphorylation, and mTOR signaling was suppressed during differentiation. Additionally, rapamycin, an mTOR 
inhibitor, increased BMD. These findings suggested that mTOR played a negative role in bone grafting mediated by osteoblasts by inhibiting the differentiation or the bone formation at late stages (30-32).

Taken together, we hypothesized that endogenous $A \beta$ may play a negative regulatory role through the mTOR signaling pathway in osteoblast precursor cells [bone marrow mesenchymal stem cells (BMSCs)] during the endogenous processing period.

It remains to be determined whether $A \beta$ plays a regulatory role in bone metabolism in $\mathrm{AD}$ patients, while the mechanisms involved also need to be identified. In the present study, we found that $A \beta$ induced osteoporotic defects through mTOR and autophagy in vitro and in vivo. $\mathrm{A} \beta$ inhibited osteoblast differentiation and activation in primary osteoblasts isolated from APP/PS1 transgenic mice. Moreover, osteoporotic changes, including low BMD and trabecular bone loss, were observed in 6-month-old APP/ PS1 mice. We further demonstrated that endogenous $A \beta$, through an mTOR-dependent inhibition of autophagy, suppressed osteoblast differentiation and osteogenic mineralization, causing osteoporotic defects in APP/PS1 mice. Our results suggested that the overactivation of mTOR by A $\beta$ might have contributed to the decrease of osteoblast activity in APP/PS1 mice. Taken together, we believe that the regulation of BMSCs by $\mathrm{A} \beta$ depended on mTOR, thereby providing a possible mechanism for the osteoporotic remodeling of $\mathrm{AD}$ patients.

We present the following article in accordance with the ARRIVE reporting checklist (available at https://dx.doi. org/10.21037/atm-21-6427).

\section{Methods}

\section{Reagents}

Antibodies recognizing A $\beta 42$ (25524-1-AP, rabbit polyclonal), beclin1 (11306-1-AP, rabbit polyclonal), and p62 (18420-1-AP, rabbit polyclonal) were purchased from Proteintech Group (Rosemont, IL, USA). The antibody detecting collagen1a (GB11022, rabbit polyclonal) was purchased from Servicebio Technology Co., Ltd. (Wuhan, China). The antibody against osteocalcin (OCN) (sc390877, rabbit polyclonal) was purchased from Santa Cruz Biotechnology (Santa Cruz, CA, USA), and the antibodies against phosphorylated S6 (p-S6) (AP0538, rabbit polyclonal), S6 (A6058, rabbit polyclonal), phosphorylated S6K (p-S6K) (AP0564, rabbit polyclonal), and S6K (A2190, rabbit polyclonal) were purchased from ABclonal Technology (Woburn, MA, USA). Affinity Biosciences (Cincinnati, OH, USA) provided antibodies detecting $\beta$-actin (T0022, rabbit polyclonal) and the goat anti-rabbit immunoglobulin $\mathrm{G}(\mathrm{IgG})$ heavy and light chain $(\mathrm{H}+\mathrm{L})$ horseradish peroxidase (HRP) (S0001, goat polyclonal). Finally, antibodies recognizing CD34 (ab198395), CD29 (ab95622), CD44 (ab112178), CD117 (ab95676), and CD45 (ab25386) were obtained from Abcam (Cambridge, MA, USA). The hematoxylin/eosin (HE) staining kit (G1120), Alizarin red S staining solution (G1452), Masson's trichrome stain kit (G1340), and Goldner trichrome stain kit (G3550) were purchased from Solarbio Life Sciences (Beijing, China). The Alkaline Phosphatase Assay Kit (P0321S) was provided by Beyotime Biotechnology (Shanghai, China).

\section{Animal experiments}

Experiments were performed under a project license (No. LAEC-2020-008) granted by Zhujiang Hospital of Southern Medical University Laboratory Animal Ethics Committee, in compliance with the Animal Welfare Policy of Zhujiang Hospital of Southern Medical University. Mice importing, transporting, housing, and breeding were conducted according to the recommendations of "The use of non-human primates in research" of The Jackson Laboratory. APP/PS1 mice (APP/PS1 group) (stock number 34829-JAX) were purchased from The Jackson Laboratory (Bar Harbor, Maine, USA). Same-age wild type littermates (WT group) were used as controls. Six mice were enrolled in each group according to previous study $(3,21,33)$. The experiments were performed with male mice to avoid potential bone changes caused by estrogen reduction in 6-month-old female mice. Grouped mice and samples were blinded as numbers when researchers were collecting and assessing data in no specific order. All mice were housed in a room with a 12-h light/dark cycle and unlimited water and food. Mice were anaesthetized by $12.5 \%$ Tribromoethanol $(0.2 \mathrm{~mL} / 10 \mathrm{~g}$ body weight) and sacrificed by overdosed.

\section{Study design}

The mice were divided into an APP/PS1 group (experimental group) and WT group (control group). Only male mice were enrolled in order to rule out the potential osteoporotic defect caused by the estrogen deficiency when female mice grow older. The behavioral changes from water maze and bone data from CT were assessed. Based 
on whether or not they were osteogenic induced (OGI), the duration of osteogenic induction, and whether or not rapamycin was added during induction, primary cells extracted were grouped into APP/PS1 (cells from APP/ PS1 mice), WT (cells from wild type mice), APP/PS1 OGI 3 days, WT OGI 3 days, APP/PS1 OGI 7 days, WT OGI 7 days, APP/PS1 OGI 3 days + rapamycin, WT OGI 3 days + rapamycin, APP/PS1 OGI 7 days + rapamycin, and WT OGI 7 days + rapamycin. Strips grayscale, area of differentiated cell and number and size of calcium deposits were assessed. All experiments were repeated three times independently to ensure relevance.

\section{Cell culture}

Femoral and tibial bones were dissected from the hind limbs of 6-mont-hold WT and APP/PS1 mice. Soft tissues and the periosteum were removed. Whole bone marrow cells were flushed out from the long bones and plated on 100 -mm tissue culture plates in $\alpha$-minimum essential medium ( $\alpha-M E M)$ containing $10 \%$ fetal bovine serum and $1 \%$ penicillin/streptomycin. Primary cells were cultured for $48 \mathrm{~h}$ at $37{ }^{\circ} \mathrm{C}$ in a humidified atmosphere of $5 \% \mathrm{CO}_{2}$. After $48 \mathrm{~h}$, the adherent cells were collected, expanded, and passaged 3 times for purification. BMSCs were identified by flow cytometry.

\section{Flow cytometry}

Marker expression was assessed using FACSCanto II flow cytometers in accordance with the manufacturer's specifications for sample preparation. The final samples were identified as BMSCs when stained positive for CD34, CD29, CD44, and CD117 and negative for CD45.

\section{Western blot assay}

Cells were lysed in radioimmunoprecipitation assay (RIPA) buffer supplemented with a protease inhibitor cocktail for $10 \mathrm{~min}$ on ice. The lysates were collected and centrifuged at $12,000 \mathrm{~g}$ for 15 minutes at $4{ }^{\circ} \mathrm{C}$. The protein concentration in the supernatant was measured using the bicinchoninic acid (BCA) assay. Next, protein lysates were mixed with loading buffer at a ratio of $1: 5$ and denatured by boiling for $5 \mathrm{~min}$. Protein lysates $(20 \mathrm{mg} /$ well) were loaded on a polyacrylamide gel for sodium dodecyl sulphatepolyacrylamide gel electrophoresis (SDS-PAGE). The proteins were transferred to polyvinylidene difluoride membranes (PVDF, Millipore, St Louis, MO, USA). The membranes were incubated with $5 \%$ skim milk for $1 \mathrm{~h}$ to block nonspecific interactions. They were then probed with specific primary antibodies against p62 $(1: 1,000)$, beclin 1 $(1: 1,000)$, collagen 1a $(1: 1,000), \mathrm{A} \beta 42(1: 1,000), \mathrm{OCN}$ $(1: 1,000)$, p-S6K $(1: 1,000)$, S6K (1:1,000), p-S6 (1:1,000), S6 $(1: 1,000)$, and $\beta$-catenin $(1: 1,000)$ overnight at $4{ }^{\circ} \mathrm{C}$. Membranes were washed in tris-buffered saline with Tween (TBST) solution (15 min) 3 times and incubated with HRP-conjugated secondary antibodies $(1: 5,000)$ for $2 \mathrm{~h}$ at room temperature. Bound antibodies were detected using an enhanced chemiluminescence detection system. The intensity of gray was quantified for each protein band using ImageJ software, and values obtained from APP/PS1 cells were normalized with values from the WT group or with values from the reference protein $\beta$-actin. Each experiment was performed in triplicate.

\section{Osteogenic induction}

For osteogenic induction, $10 \mathrm{nM}$ of dexamethasone, $50 \mathrm{mg} / \mathrm{mL}$ of ascorbic acid 2-phosphate, and $10 \mathrm{mM}$ of $\beta$-glycerophosphate were added to the $\alpha$-MEM culture medium. Rapamycin (Sigma-Aldrich, St Louis, MO, USA) was added to the culture medium as stated in the results section.

\section{Alizarin red $S$ staining}

Osteogenic induced BMSCs were washed twice with phosphate-buffered saline (PBS) and fixed in $4 \%$ paraformaldehyde for $30 \mathrm{~min}$. Samples were washed with deionized water and incubated with $1 \%$ Alizarin red S solution ( $\mathrm{pH} 4.2)$ for $1 \mathrm{~h}$ at room temperature. The plates were scanned and observed under an optical microscope. The calcium deposits stained in orange and red were identified and quantified in each group. For quantification, the Alizarin red S-stained deposits were incubated with 10\% cetylpyridinium chloride (Sigma-Aldrich) for $30 \mathrm{~min}$. The mixture was then placed in 96-well plates and absorbance detected at $620 \mathrm{~nm}$ in a microplate reader. Experiments were performed in triplicate.

\section{Preparation of decalcified sections}

The harvested hind limbs were washed twice with normal saline, fixed in $4 \%$ paraformaldehyde at room temperature for $48 \mathrm{~h}$, and decalcified in ethylenediaminetetraacetic acid 
(EDTA) for 4 weeks. The samples were dehydrated in a graded series of ethanol solutions, embedded in paraffin, and sectioned to obtain sagittal sections ( $4 \mu \mathrm{m}$ thick).

\section{HE and Masson's trichrome staining}

The sections were stained with HE for morphological analysis. Cell nuclei were stained in blue by hematoxylin, and the extracellular matrix was stained in pink by eosin. Bones appeared as deep pink/red. Images of stained sections were acquired under a light microscope to evaluate differences in trabecular bone tissue between WT and APP/PS1 mice.

For Masson's trichrome staining, the sections were processed following the kit's instructions.

\section{Goldner's trichrome staining}

After dissection, the femurs were fixed in $4 \%$ paraformaldehyde for $24 \mathrm{~h}$. They were then dehydrated through a graded series of ethanol (70-100\%) and xylene before being embedded in methyl methacrylate (MMA) without prior decalcification. Five-micrometer-thick sections were processed for Goldner's trichrome staining according to the manufacturer's instruction.

\section{Morris water maze}

The water maze apparatus (a 120-cm tank) was filled with room temperature water $\left(25^{\circ} \mathrm{C}\right)$, which was made opaque by the addition of nontoxic, white tempera paint. The water temperature was monitored using a thermometer before beginning the trials. Several high-contrast visual cues were placed around the apparatus in the room. Mice were placed on the platform for $10 \mathrm{~s}$ prior to the first training trial of each day to reduce stress. Over a period of 4 days, mice were trained to swim to a 14-cm diameter circular clear Plexiglas platform submerged $1.5 \mathrm{~cm}$ beneath the surface of the water and invisible to the mice while swimming. If they did not find the platform within the 88-s trial limit, they were guided to the hidden platform. The platform location was kept in quadrant III throughout the training. For each trial, the mouse was placed into the tank at 1 of 3 designated start points in a semirandom order. To ensure that memory differences were not due to lack of task learning, each mouse underwent daily 4-trial sessions ( $88 \mathrm{~s}$ each with a 1-min interval).

Twenty-four hours after the final training trial, spatial memory retention was assessed in a probe trial consisting of an 88-s free swim in the pool without the platform.
An overhead-mounted video tracking system was used to monitor and quantify different parameters during the training and probe trials. The time spent and distance travelled in each quadrant and the number of times crossing the platform location were measured, with the results compared by ANOVA. The following day, the mice underwent a visible platform test to ensure no physical impairment influenced the performance during the test (data not shown). The parameters were the same as on the training days, except that the location of the platform was indicated by a visible flag placed on it. The platform was placed at a different location than that during the probe trial to avoid any spatial memory-related success.

\section{Microcomputed tomography (micro-CT)}

Quantitative analysis of mouse femora was performed on a micro-CT scanner (Viva CT40; SCANO Medical AG, Bassersdorf, Switzerland) at $12 \mu \mathrm{m}$ resolution. Scanning was performed at the distal end of the femur and was extended proximally for 300 slices. We started the morphometric analysis with the first slice in which the femoral condyles were fully merged and extended proximally for 100 slices. We manually segmented the trabecular bone from the cortical shell on key slices using a contouring tool and morphed the contours automatically to segment the trabecular bone on all slices. A 3-dimensional structure and morphometry were constructed and analyzed for bone volume/tissue volume fraction (BV/TV, \%), BMD (mg $\mathrm{HA} / \mathrm{mm}^{3}$ ), trabecular number $\left(\mathrm{Tb} . \mathrm{N}, \mathrm{mm}^{-1}\right)$, trabecular thickness (Tb.Th, mm), and trabecular separation (Tb.Sp, $\mathrm{mm})$. We also conducted micro-CT imaging in the middiaphysis of the femur and performed midshaft evaluation of 100 slices to quantify the cortical thickness, BMD, and outer/inner perimeter of the midshaft. And a reduction of BV/TV, BMD, Tb.N and Tb.Th and elevation of Tb.Sp can indicate osteoporotic defect.

\section{$R N A$ isolation and quantitative real-time PCR (qPCR)}

Messenger RNA (mRNA) expression was analyzed by qPCR. Total RNA was extracted from cell samples using the TRIzol extraction method. After assessing the purity and concentration of the RNA, it was reverse transcribed to cDNA using PrimeScript RT Master Mix (Perfect Real-Time; Takara Bio Inc., Kusatsu, Shiga, Japan). Realtime PCR amplification was performed using TB Green Premix Ex Taq II (Tli RNaseH Plus; Takara). The mRNA 
Table 1 Primer sequences

\begin{tabular}{lcc}
\hline Primers & Forward 5'-3' & Reverse 5'-3' $^{\prime}$ \\
\hline Beclin1 & ATGGAGGGGTCTAAGGCGTC & TCCTCTCCTGAGTTAGCCTCT \\
APP & TCCGAGAGGTGTGCTCTGAA & CCACATCCGCCGTAAAAGAATG \\
OCN & GAAGCCCAGCGGTGCA & CACTACCTCGCTGCCCTCC \\
$\beta$-actin & GGCTGTATTCCCCTCCATCG & CCAGTTGGTAACAATGCCATGT \\
\hline
\end{tabular}

expression levels were normalized to the expression level of the housekeeping gene glyceraldehyde 3-phosphate dehydrogenase (GAPDH). Relative gene expression levels were calculated using the $2^{-\Delta \Delta \mathrm{Ct}}$ method. Each experiment was performed in triplicate. The primers were used as shown in Table 1.

\section{Immunofluorescence staining and confocal imaging analysis}

Cells were plated on a confocal dish and fixed with $4 \%$ paraformaldehyde for $30 \mathrm{~min}$, permeabilized with $0.1 \%$ v/v Triton X-100/1× PBS (10 min), and blocked with $10 \%$ normal goat serum prior to incubation with primary antibodies (rabbit anti-A $342,1: 100$; Proteintech) overnight. Cells were then labeled with the appropriate secondary antibody raised in goat (1:150; Thermo Fisher Scientific, Waltham, MA, USA). Images were taken using the LSM 800 confocal microscope (Zeiss; Oberkochen, Germany), and excitation and acquisition parameters were constrained across all paired comparisons.

\section{Statistical analysis}

All assays were repeated for 3 independent experiments. All data of different groups of animals, cells or strips are presented as mean \pm standard error of mean (SEM). Significance was determined by one-way ANOVA, followed by Bonferroni post hoc test for multiple comparisons using the GraphPad Prism 8.0 software (GraphPad Software Inc., La Jolla, CA, USA). The significance threshold was set at $\mathrm{P}<0.05$.

\section{Results}

\section{Cognitive impairments in APP/PS1 mice}

As Morris water maze (MWM) performance is hippocampusdependent, it was used to evaluate the cognitive function of APP/PS1 mice. After the confirmation of relative even of weight, size and intact of coat of each group of mice.
We assessed the spatial learning and reference memory of 6-month-old WT and APP/PS1 mice. In our MWM test, platform position training was performed during the first 4 days with a hidden platform submerged $2 \mathrm{~cm}$ in water. The probe test was carried out on the fifth day. Data from each group of six mice are taken into analysed.

The time to reach the submerged platform was recorded for WT and APP/PS1 mice during the 4 days of navigation training. The average escape latency of the APP/PS1 group in the last 3 days of training showed a significant increase when compared with the WT group $(\mathrm{P}<0.01$; Figure $1 A)$. Representative paths followed by the mice on the fourth day of training are shown in Figure 1B. On the fifth day of the MWM test, the platform was removed to perform the probe test. The average time spent and the average swimming distance in the target quadrant as well as the number of times crossing the platform location were measured and recorded for later comparison. Our results revealed that time spent in the target area and swimming distance within this area differed greatly between the WT and APP/PS1 groups. Representative images of the swimming paths for both groups in the probe test are shown in Figure 1C. APP/PS1 mice spent significantly less time and swam shorter distances in the target quadrant to search for the platform compared with the WT mice $(\mathrm{P}<0.01$; Figure 1D,1E). Moreover, the APP/PS1 mice crossed the platform location less frequently compared with the WT group $(\mathrm{P}<0.01$; Figure $1 F)$. Our results demonstrated that the APP/PS1 mice had impaired spatial learning and memory compared with WT mice.

\section{Endogenous Aß induced osteoporotic defects in mice}

To evaluate bone changes, APP/PS1 and WT mice were subjected to micro-CT scans. Data from each group of six mice are all included in analysis. Representative reconstructed 3D images of trabecular bone showed that APP/PS1 mice had thinner and fewer trabeculae compared with WT mice (Figure $2 A$ ). In the APP/PS1 group, the average $\mathrm{BMD}$ was significantly lower than that for the WT 

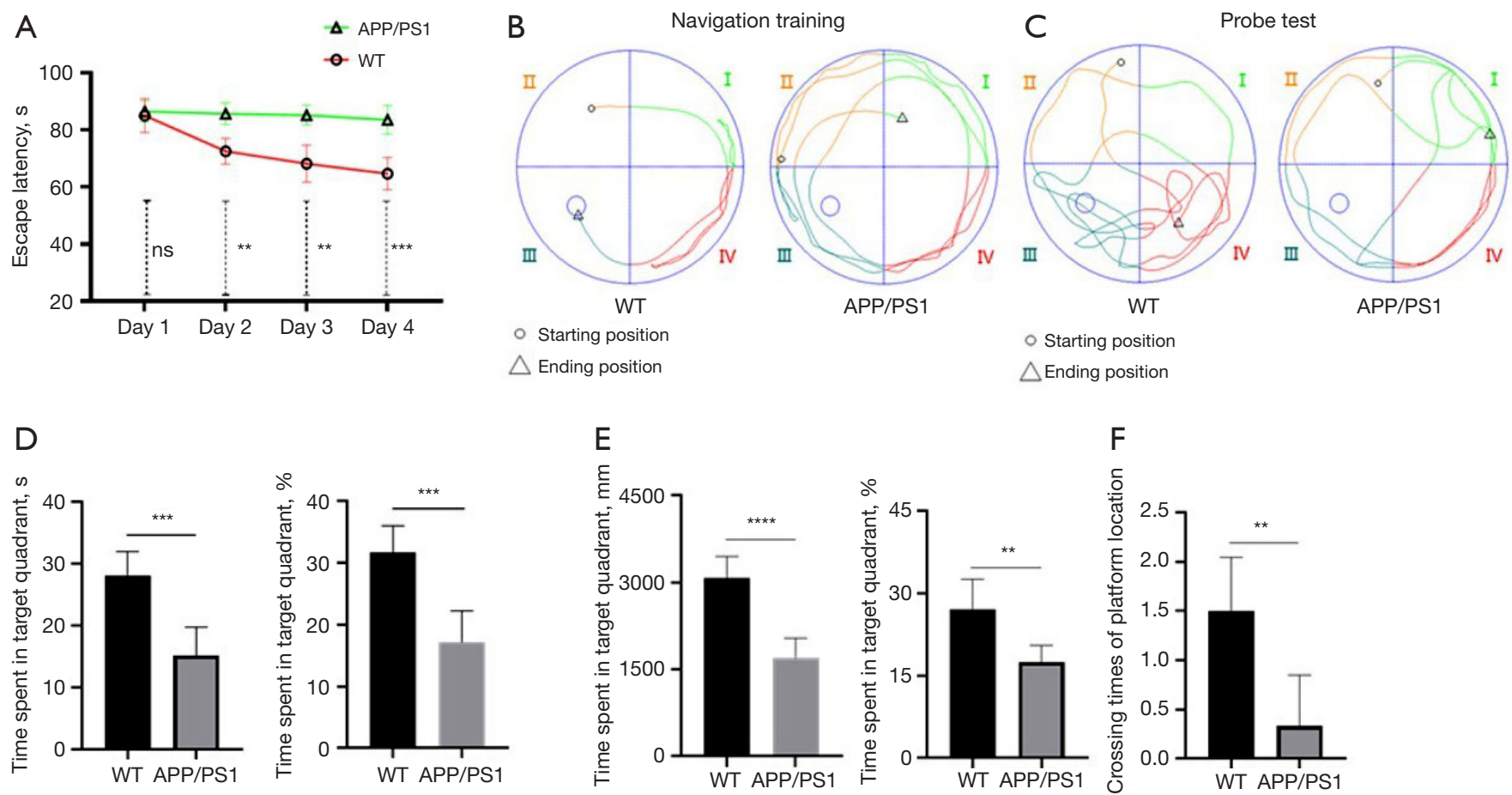

Figure 1 Cognitive impairments in APP/PS1 mice. (A) Changes of escape latency during training days; (B) typical routes on 4th training day; (C) typical routes in probe test; (D) searching time and proportion in target area; (E) swimming distance and proportion in the target area; (F) platform crossing times. ns, no significance; ${ }^{* *} \mathrm{P}<0.01 ;{ }^{* * *} \mathrm{P}<0.001 ;{ }^{* * * *} \mathrm{P}<0.0001$.

mice (Figure $2 B$ ). The BV/TV value range was between 0.1 and 0.15 for the APP/PS1 group, whereas it was smaller (between 0.15 and 0.2) for WT mice (Figure 2C). Similarly, the Conn. D in the APP/PS1 group was decreased compared to the average value for WT mice (Figure 2D). Additional differences were observed mostly for trabecular bone, with the Tb.N, Tb.Sp, and Tb. Th decreased in APP/PS1 mice (Figure 2E-2G). The osteoporotic effects of intracellular $A \beta$ were mild in cortical bone and affected mostly cancellous bone. Therefore, we suspected that endogenous $\mathrm{A} \beta$ might have affected bone metabolism by interfering with the function of cells from the bone marrow cavity or from trabeculae.

\section{Endogenous Aß induced trabecular deficit in APP/PS1 mice}

To observe the effects of endogenous $A \beta$ on bone formation, histomorphometrical analyses were conducted, including HE staining, Goldner's trichrome staining, and Masson staining. HE staining showed that the distal femur of APP/ PS1 mice possessed fewer and thinner trabecular bones and larger gaps between trabecular bones compared with femurs of WT mice (Figure 3A). Masson staining revealed that newly formed collagen (blue staining) and the resultant mature bone (red staining) were reduced in the trabeculae of the distal femur in APP/PS1 mice (Figure 3B). This suggested that APP/PS1 mice had a lower level of collagen synthesis, which is directly related to cells responsible for collagen composition, such as BMSCs and osteoblasts. Goldner's trichrome staining showed that the amount and thickness of the mineralized trabeculae (green area) of the distal femur were significantly reduced in APP/PS1 mice compared to those in WT mice (Figure 3C). Both groups had little osteoid and cartilage (red and purple) considering their age (6 months). Taken together, these results suggested that endogenous $A \beta$ had an inhibitory effect on bone grafting in APP/PS1 mice, especially in trabeculae. This inhibitory effect might have depended mainly on cells responsible for collagen synthesis, such as BMSCs and osteoblasts.

\section{The differentiation and ossification of BMSCs from APP/ PS1 mice are inbibited by endogenous A $\beta$}

We then extracted BMSCs from WT and APP/PS1 mice. After cell extraction, the presence of positive surface markers (CD34, CD29, CD44, and CD117) and the 

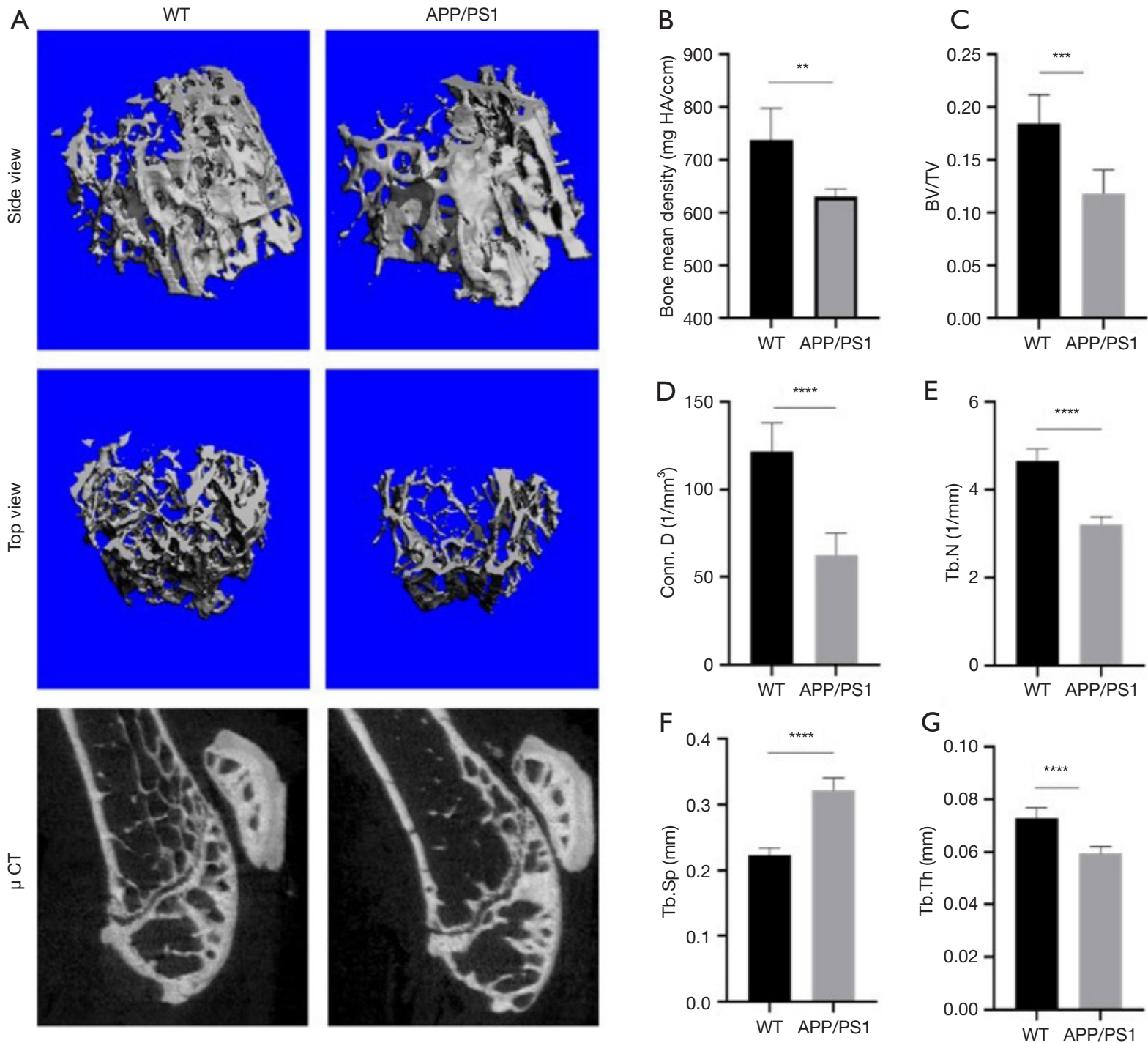

Figure 2 Endogenous $\mathrm{A} \beta$ induced osteoporotic defect in mice. (A) Representative pictures of 3D reconstruction of trabeculae; (B) bone mineral density (BMD); (C) bone volume/tissue volume (BV/TV) ratio; (D) bone connection density (Conn. D); (E) the number of trabecular bone (Tb.N); (F) trabecular bone separation (Tb.Sp); (G) trabecular bone thickness (Tb. Th). ${ }^{* *} \mathrm{P}<0.01 ;{ }^{* * *} \mathrm{P}<0.001 ;{ }^{* * * *} \mathrm{P}<0.0001$.

absence of negative markers (CD45) for BMSCs were confirmed by flow cytometry to ensure cell purity for later experiments. The purity of the cells extracted was more than $95 \%$ (Figure $4 A$ ). We analyzed the capacity of BMSCs for osteogenic differentiation and ossification in vitro. We detected the activity of alkaline phosphatase (ALP), a marker of BMSC differentiation, using a staining kit after 7 days of OGI. The staining in APP/PS1 BMSCs was lighter and the stained area was smaller than that in the WT group (Figure 4B). Thus, the ALP activity of BMSCs isolated from APP/PS1 mice was significantly reduced compared to the ALP activity in WT BMSCs. Osteogenesis was assessed by measuring mineralization ability using Alizarin red S staining in BMSCs of WT and APP/PS1 mice after 7 days of OGI. More and larger calcium nodules were observed in BMSCs from WT mice than in BMSCs from APP/PS1 mice, suggesting that intracellular $A \beta$ significantly inhibited the mineralization ability of BMSCs and thereafter hinder osteogenesis (Figure $4 C$ ). The distribution of amyloid $\beta 1-42$ (A $\beta 42)$ was investigated by immunofluorescence analysis and laser confocal scanning, which confirmed the intracellular expression and accumulation of $\mathrm{A} \beta$ in BMSCs of APP/PS1 mice (Figure $4 D$ ). Taken together, these findings suggested that the accumulation of endogenous $A \beta$ decreased the 

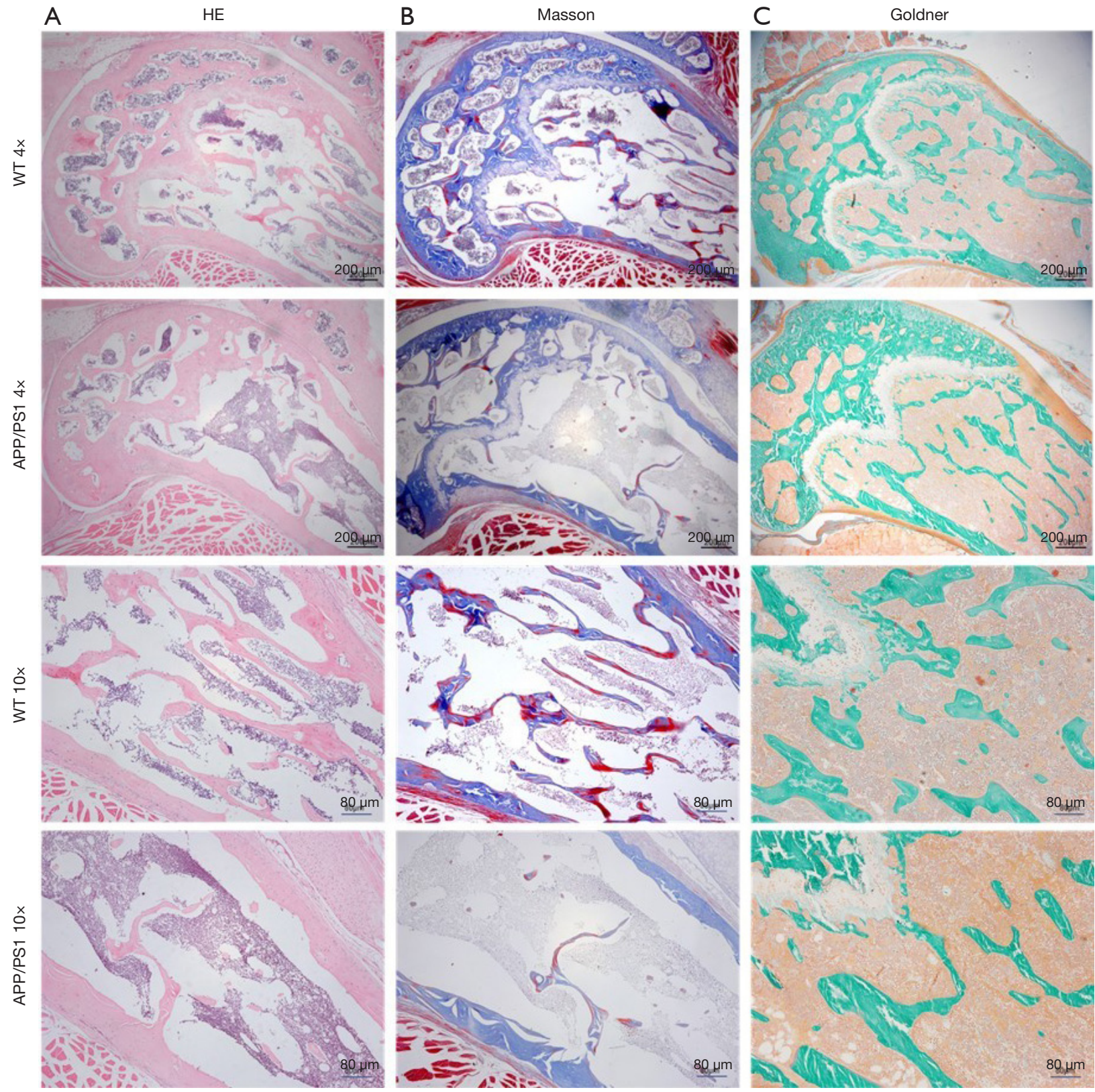

Figure 3 Endogenous A $\beta$ induced trabecular deficit in APP/PS1 mice. (A) Hematoxylin/eosin (HE) staining of the distal femur of mice; (B) Masson staining of distal femur (blue for collagen, red for mature fiber); (C) Goldner's trichrome staining (green for mineralized bone).

differentiation potential and suppressed the ossification capability of BMSCs, thus leading to osteoporotic deficit.

\section{Endogenous Aß inbibited osteogenesis and autophagy in BMSCs}

Previous studies have revealed that $\mathrm{A} \beta 42$ may affect cell autophagy in neurons (34-36), and defective autophagy has been reported in $\mathrm{AD}$ (37). Autophagy has also been found to be linked to osteogenesis (38-40). Therefore, we suspected that $A \beta$ was able to regulate osteogenesis by altering autophagy in BMSCs. To verify this hypothesis, we compared the mRNA expression of the osteogenic markers OCN and collagen $1 \mathrm{a}$, the autophagic marker beclin1, and APP in BMSCs in normal culture medium. Endogenous $A \beta$ significantly downregulated transcriptional 

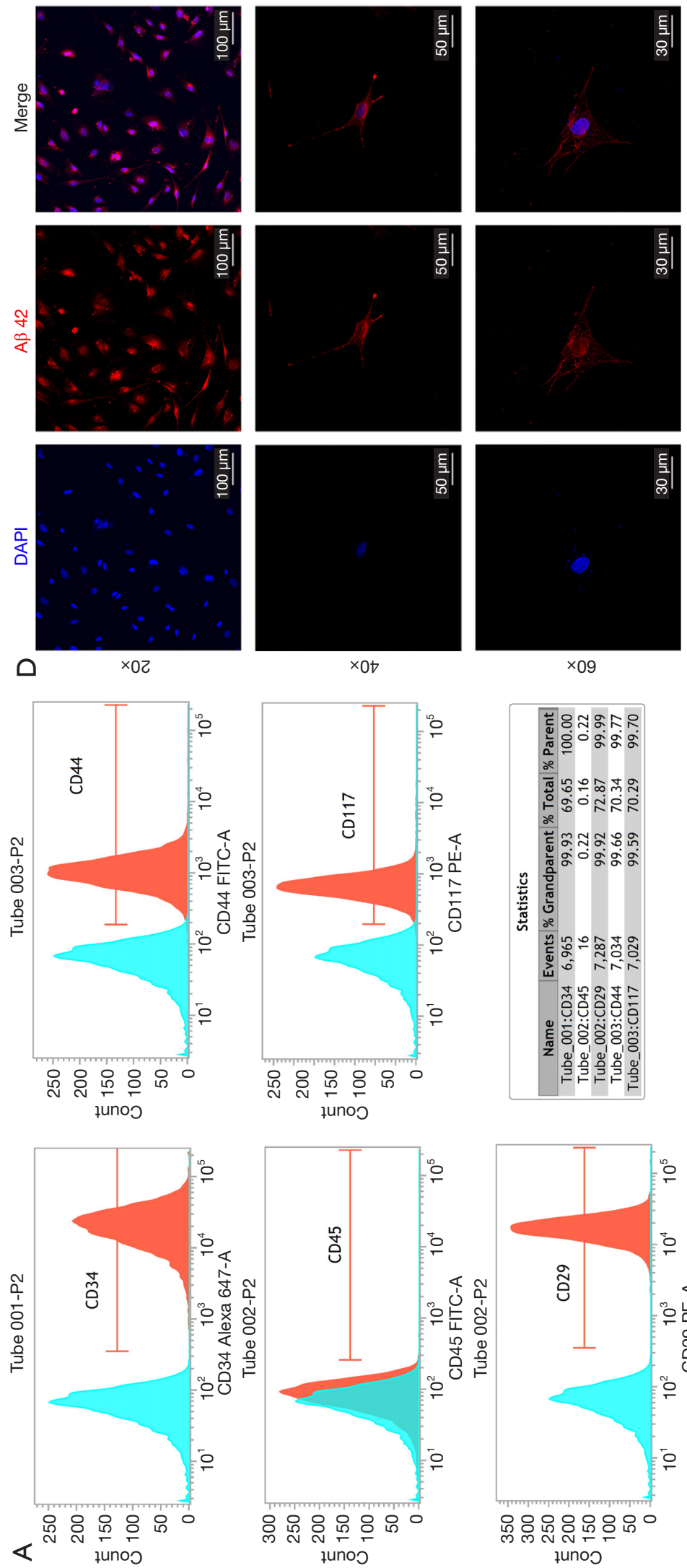

$\times 09$
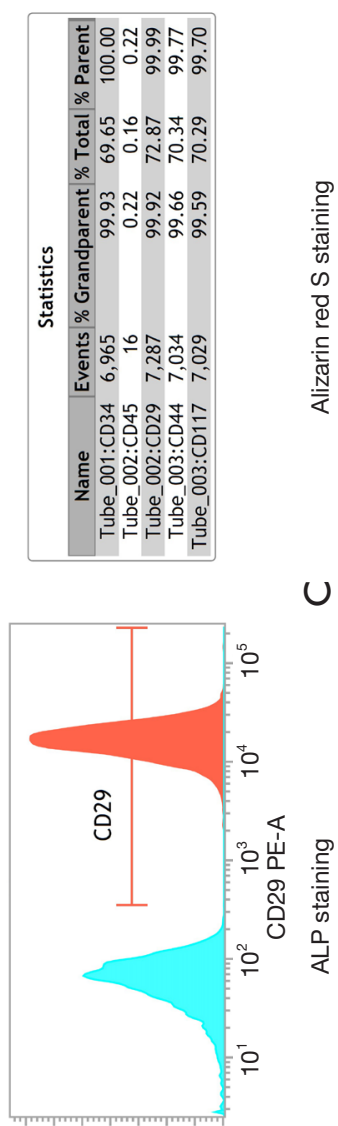

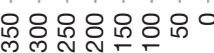
tuno?
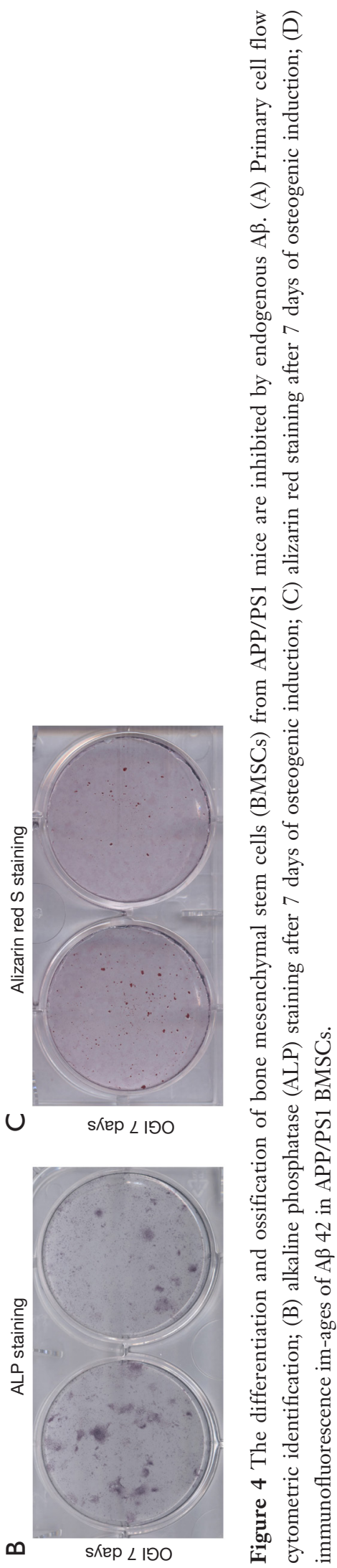

(c) Annals of Translational Medicine. All rights reserved. 

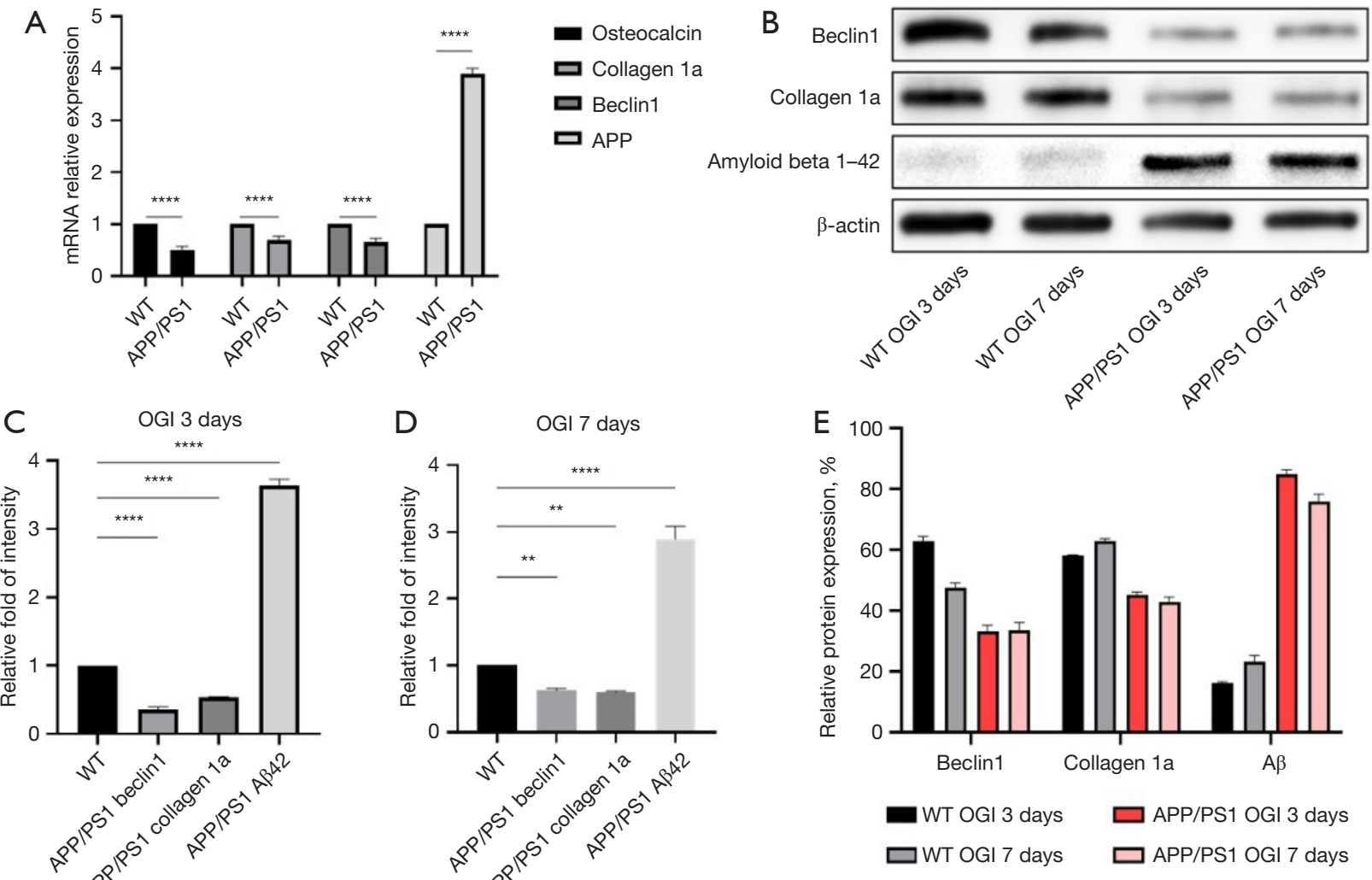

Figure 5 Endogenous $A \beta$ inhibited osteogenesis and autophagy in bone mesenchymal stem cells (BMSCs). (A) The effect of endogenous A $\beta$ on the mRNA transcription level of BMSCs; (B) changes of autophagic and osteogenic protein expression after osteogenic in-duction (OGI); $(\mathrm{C}, \mathrm{D})$ relative protein expression of $\mathrm{APP} / \mathrm{PS} 1$ to wild type (WT); (E) relative protein expression of target proteins to $\beta$-actin. ${ }^{* *} \mathrm{P}<0.01$; **** $\mathrm{P}<0.0001$.

levels of autophagy and osteogenesis markers, and APP was significantly increased in APP/PS1 cells (Figure 5A). Further, the osteogenic changes in BMSCs were confirmed by western blot. The expression levels (represented by strip grayscale) of A 342 in BMSCs from APP/PS1 mice were consistently higher than the A $\beta 42$ levels in WT cells (Figure $5 B$ ). After 3 and 7 days of OGI, A $\beta$ significantly decreased collagen $1 \mathrm{a}$ and beclin1 protein levels when compared to WT samples (Figure $5 C, 5 D)$ or normalized with the reference protein, $\beta$-actin (Figure 5E). All differences were statistically significant $(\mathrm{P}<0.001)$. These results indicated that endogenous $\mathrm{A} \beta$ might have inhibited autophagy in BMSCs.

\section{Endogenous Aß suppresses late-stage osteogenesis of BMSCs through the mTOR-dependent inhibition of autophagy}

The mTOR signaling pathway is a major regulator of autophagy in cells $(41,42)$. Emerging evidence has demonstrated that the mTOR signaling pathway plays a critical role in the regulation of osteogenic differentiation and ossification of BMSCs (43-45). Thus, the mTOR signaling pathway might be involved in the pathological inhibition of osteogenesis and autophagy by endogenous $\mathrm{A} \beta$ in APP/PS1 BMSCs. Therefore, we investigated the activation of the mTOR pathway in APP/PS1 BMSCs. After 3 and 7 days of OGI, we treated APP/PS1 and WT BMSCs with or without $50 \mathrm{~nm}$ of rapamycin, an mTOR inhibitor and autophagy activator (42). We performed western blot of $\mathrm{OCN}$ and collagen1a as markers of late-stage osteogenesis and calcium deposition; beclin1 and p62 as hallmarks of cell autophagy; and p-S6, S6, p-S6K, and S6K as measures of the activation levels of the mTOR pathway (Figure $6 A$ ). We detected the band intensities (Figure $6 B$ ) and compared the intensity ratios of p-S6 to $\mathrm{S} 6$ and $\mathrm{p}-\mathrm{S} 6 \mathrm{~K}$ to $\mathrm{S} 6 \mathrm{~K}$ to assess mTOR signaling activation (Figure 6C). A $\beta$ was consistently overexpressed in APP/PS1 mice, treated with rapamycin or not, compared to the WT group (Figure 6A). The phosphorylation levels of S6 and S6K were noticeably 
higher in the APP/PS1 group compared to the WT group (Figure 6A). These increases in p-S6-to-S6 and p-S6K-toS6K ratios were eradicated by rapamycin, which confirmed the effective inhibition of mTOR activation (Figure 6C). The expression of late-stage osteogenesis markers (OCN and collagen 1a) and autophagy markers (beclin1 and p62) were downregulated in APP/PS1 mice compared to the WT group (Figure $6 A, 6 B$ ). However, the inhibition of mTOR by rapamycin significantly restored the expression levels of OCN, collagen1a, beclin1, and p62 in APP/PS1 BMSCs (Figure 6A-6C). ALP and Alizarin red S staining further confirmed the inhibitory effect of $\mathrm{A} \beta$ on late-stage osteogenesis and its reversal by rapamycin (Figure 6D,6E). ALP staining was lighter and the staining areas were smaller in APP/PS1 BMSCs than in WT BMSCs (Figure $6 D)$. After the addition of rapamycin, ALP staining was similar in the APP/PS1 and WT groups. The formation of calcium nodules stained by Alizarin red S was significantly suppressed at each time interval in the APP/PS1 group (Figure $6 E$ ). Therefore, the addition of rapamycin in the culture medium effectively rescued the inhibition of the ossification caused by endogenous A $\beta$ in APP/PS1 BMSCs. These results suggested that endogenous $A \beta$ suppressed late-stage osteogenic processes by inhibiting autophagy in an mTOR-dependent manner.

\section{Discussion}

In this study, we showed the role of endogenous $A \beta$ in bone grafting and provided a link between osteoporotic deficit in $\mathrm{AD}$ and $\mathrm{A} \beta$. We found that in the BMSCs extracted from APP/PS1 transgenic mice, A $\beta$ suppressed autophagy by activating the mTOR pathway and inhibited osteogenesis, especially calcium deposition. Ultimately, this led to bone loss in the AD mouse model.

We confirmed the simultaneous occurrence of dementia and osteoporotic deficit in vivo in the APP/PS1 transgenic mouse model. We also provided evidence regarding the mechanisms underlying the bone loss in $\mathrm{AD}$. However, the link between $\mathrm{AD}$ and $\mathrm{OP}$ still needs to be validated in humans.

Our results constitute the first description of an inhibitory role of endogenous $A \beta$ on bone formation involving BMSCs. We showed that endogenous $\mathrm{A} \beta$ might have inhibited osteogenesis by suppressing late-stage bone formation in BMSCs and reducing calcium deposition. Our findings are compatible with some previous studies $(3,33)$. However, a negative role for $A \beta$ in bone metabolism has also been described. In particular, it was shown that
$A \beta$ promoted bone formation and enhanced bone volume $(20,21)$. The differences between our findings and those of previous studies might have been related to experimental design as the previous studies had used exogenous $A \beta$ injected into mice to induce $\mathrm{AD}$-like pathology. In light of organism complexity and the precise dosage to be given to mice, we decided to use a transgenic mouse line producing endogenous $A \beta$. We were then able to simulate the pathological processes induced by excessive deposition of $A \beta$ generated from the cleavage of APP. Accumulated A $\beta$ is originally produced from APP in the endoplasmic reticulum and then goes through the Golgi apparatus and lysosomal/ endosomal transport pathways before finally being released to the cytosol or extracellular matrix (46). Injection of exogenous $A \beta$ circumvents the process of $A \beta$ production and deposition inside the cells and potentially overlooks the effects of these processes on the organism.

We focused on studying the mechanisms induced by $\mathrm{A} \beta$ in BMSCs. Previous investigations of $\mathrm{A} \beta$ effects on bone turnover in osteoclasts and osteoblasts involved several pathways affected by $A \beta$, including the I $\mathrm{B}-\alpha / \mathrm{ERK}$ and OPG/RANKL/RANK systems in osteoclasts and Wnt/ $\beta$ catenin signaling in osteoblasts $(20,21)$. Our study revealed for the first time a link between $A \beta$ and the mTOR pathway in BMSCs by confirming that endogenous $A \beta$ promoted the activation of mTOR signaling and subsequently suppressed bone formation by BMSCs. We assumed that the inhibition of autophagy due to the overactivation of mTOR was the main cause of the osteogenesis loss. Thus, when it comes to bone metabolism, $\mathrm{A} \beta$ in $\mathrm{AD}$ patients is supposed to produce from APP in the endoplasmic reticulum and through the Golgi apparatus and lysosomal/endosomal assembling in BMSCs, and during the intracellular processing, $\mathrm{A} \beta$ can activate mTOR signaling pathway in BMSCs after which the activation leads to a inhibition of autophagy and causes the impair of differentiation and calcium deposit of BMSCs, ultimately induces the osteoporotic defect in $\mathrm{AD}$ patients. The molecular processes involved in the suppression of osteogenesis by $\mathrm{A} \beta$-mediated inhibition of autophagy need to be further characterized. In particular, the use of tools to modulate autophagy while bypassing the mTOR pathway will confirm the role of autophagy in the effects of $A \beta$ on osteogenesis. The mechanisms of $\mathrm{A} \beta$ and mTOR interaction also remain unclear.

\section{Conclusions}

In summary, our results indicated that endogenous $A \beta$ 

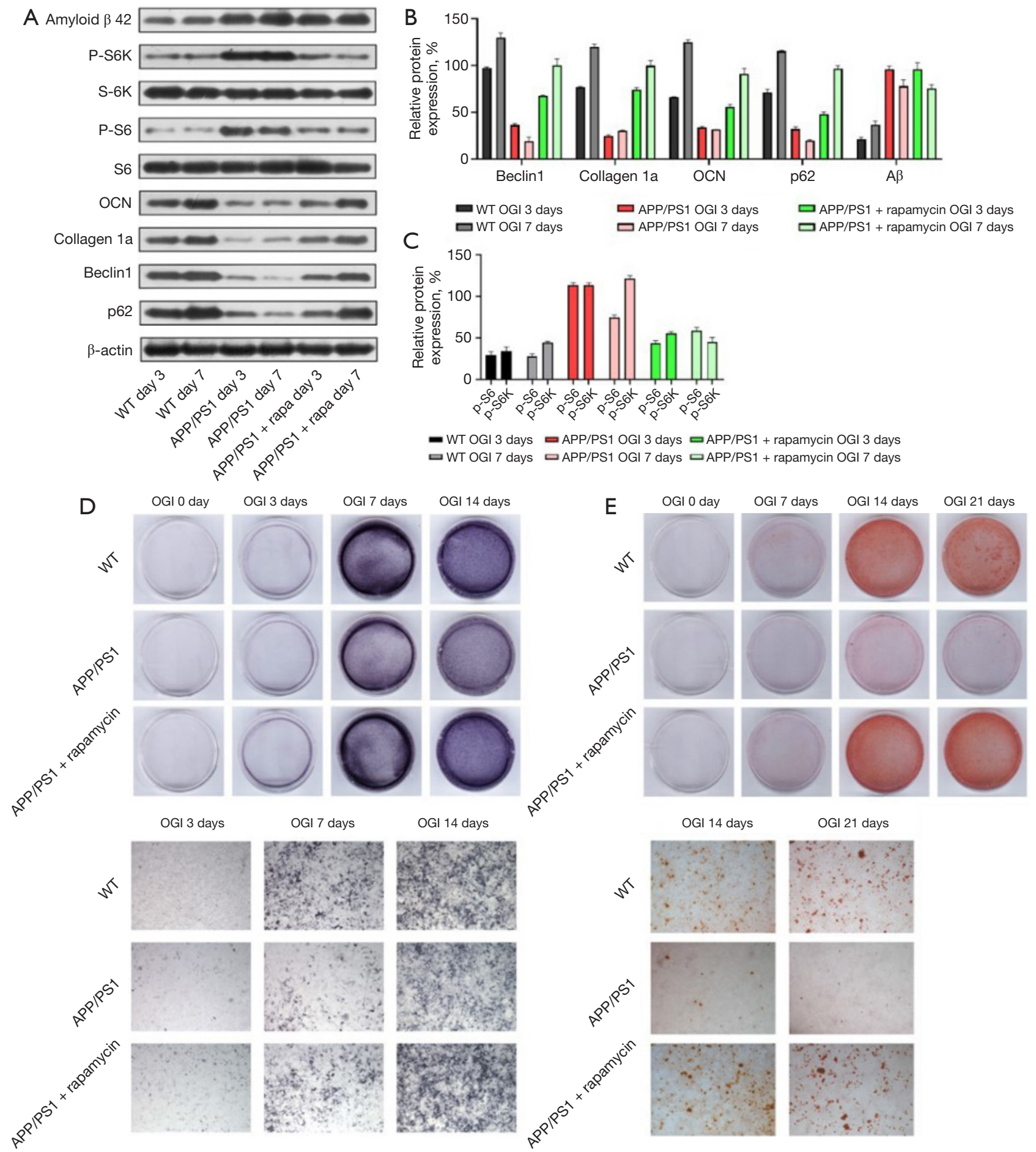

Figure 6 Endogenous A $\beta$ suppresses late-stage osteogenesis of bone mesenchymal stem cells (BMSCs) through the mammalian target of rapamycin (mTOR)-dependent inhibition of autophagy. (A) Representative protein strips; (B) relative protein expression; (C) relative expression of phosphorylated S6K (p-S6K) and phosphorylated-S6 (p-S6); (D) scanned and 4x microscopic images of alkaline phosphatase (ALP) staining; (E) scanned and $4 \times$ microscopic images of alizarin red staining. 
significantly suppressed bone formation by BMSCs. The activation of mTOR signaling by $A \beta$ is an indispensable part of this regulation, suggesting that autophagy was involved in the process. These findings provide a novel insight into the mechanism of endogenous $A \beta$ in BMSCs and the relationship between $\mathrm{AD}$ and $\mathrm{OP}$.

\section{Acknowledgments}

Funding: This study was financially supported by the Natural Science Foundation of Guangdong Province, China (2018A0303130242, 2021A1515010005) and Presidential Foundation of the Third Affiliated Hospital of Southern Medical University, China (YM2021006).

\section{Footnote}

Reporting Checklist: The authors have completed the ARRIVE reporting checklist. Available at https://dx.doi. org/10.21037/atm-21-6427

Data Sharing Statement: Available at https://dx.doi. org/10.21037/atm-21-6427

Conflicts of Interest: All authors have completed the ICMJE uniform disclosure form (available at https://dx.doi. org/10.21037/atm-21-6427). All authors report funding from Natural Science Foundation of Guangdong Province, China. The authors have no other conflicts of interest to declare.

Ethical Statement: The authors are accountable for all aspects of the work in ensuring that questions related to the accuracy or integrity of any part of the work are appropriately investigated and resolved. Experiments were performed under a project license (No. LAEC-2020008) granted by Zhujiang Hospital of Southern Medical University Laboratory Animal Ethics Committee, in compliance with the Animal Welfare Policy of ZhuJiang Hospital of Southern Medical University.

Open Access Statement: This is an Open Access article distributed in accordance with the Creative Commons Attribution-NonCommercial-NoDerivs 4.0 International License (CC BY-NC-ND 4.0), which permits the noncommercial replication and distribution of the article with the strict proviso that no changes or edits are made and the original work is properly cited (including links to both the formal publication through the relevant DOI and the license). See: https://creativecommons.org/licenses/by-nc-nd/4.0/.

\section{References}

1. Dengler-Crish CM, Elefteriou F. Shared mechanisms: osteoporosis and Alzheimer's disease? Aging (Albany NY) 2019;11:1317-8.

2. Bhandari M, Swiontkowski M. Management of Acute Hip Fracture. N Engl J Med 2017;377:2053-62.

3. Xia WF, Jung JU, Shun C, et al. Swedish mutant APP suppresses osteoblast differentiation and causes osteoporotic deficit, which are ameliorated by N-acetyl-Lcysteine. J Bone Miner Res 2013;28:2122-35.

4. Weller II. The relation between hip fracture and alzheimer's disease in the canadian national population health survey health institutions data, 1994-1995. A crosssectional study. Ann Epidemiol 2000;10:461.

5. Weller I, Schatzker J. Hip fractures and Alzheimer's disease in elderly institutionalized Canadians. Ann Epidemiol 2004;14:319-24.

6. Loskutova N, Honea RA, Vidoni ED, et al. Bone density and brain atrophy in early Alzheimer's disease. J Alzheimers Dis 2009;18:777-85.

7. Loskutova N, Honea RA, Brooks WM, et al. Reduced limbic and hypothalamic volumes correlate with bone density in early Alzheimer's disease. J Alzheimers Dis 2010;20:313-22.

8. Zarrouk A, Vejux A, Mackrill J, et al. Involvement of oxysterols in age-related diseases and ageing processes. Ageing Res Rev 2014;18:148-62.

9. Long JM, Holtzman DM. Alzheimer Disease: An Update on Pathobiology and Treatment Strategies. Cell 2019;179:312-39.

10. Scheltens P, Blennow K, Breteler MM, et al. Alzheimer's disease. Lancet 2016;388:505-17.

11. Zhang $\mathrm{H}, \mathrm{Ma} \mathrm{Q}$, Zhang YW, et al. Proteolytic processing of Alzheimer's $\beta$-amyloid precursor protein. J Neurochem 2012;120 Suppl 1:9-21.

12. Dengler-Crish CM, Smith MA, Wilson GN. Early Evidence of Low Bone Density and Decreased Serotonergic Synthesis in the Dorsal Raphe of a Tauopathy Model of Alzheimer's Disease. J Alzheimers Dis 2017;55:1605-19.

13. Yang MW, Wang TH, Yan PP, et al. Curcumin improves bone microarchitecture and enhances mineral density in APP/PS1 transgenic mice. Phytomedicine 2011;18:205-13.

14. Cui S, Xiong F, Hong Y, et al. APPswe/A $\beta$ regulation of 
osteoclast activation and RAGE expression in an agedependent manner. J Bone Miner Res 2011;26:1084-98.

15. Jankowsky JL, Fadale DJ, Anderson J, et al. Mutant presenilins specifically elevate the levels of the 42 residue beta-amyloid peptide in vivo: evidence for augmentation of a 42-specific gamma secretase. Hum Mol Genet 2004;13:159-70.

16. Reiserer RS, Harrison FE, Syverud DC, et al. Impaired spatial learning in the APPSwe + PSEN1DeltaE9 bigenic mouse model of Alzheimer's disease. Genes Brain Behav 2007;6:54-65.

17. Jankowsky JL, Slunt HH, Ratovitski T, et al. Coexpression of multiple transgenes in mouse CNS: a comparison of strategies. Biomol Eng 2001;17:157-65.

18. Compston JE, McClung MR, Leslie WD. Osteoporosis. Lancet 2019;393:364-76.

19. Seeman E, Delmas PD. Bone quality--the material and structural basis of bone strength and fragility. N Engl J Med 2006;354:2250-61.

20. Li S, Yang B, Teguh D, et al. Amyloid $\beta$ Peptide Enhances RANKL-Induced Osteoclast Activation through NF- $\beta$ B, ERK, and Calcium Oscillation Signaling. Int J Mol Sci 2016;17:1683.

21. Yang B, Li S, Chen Z, et al. Amyloid $\beta$ peptide promotes bone formation by regulating $\mathrm{Wnt} / \beta$-catenin signaling and the OPG/RANKL/RANK system. FASEB J 2020;34:3583-93.

22. Saxton RA, Sabatini DM. mTOR Signaling in Growth, Metabolism, and Disease. Cell 2017;169:361-71.

23. Murugan AK. mTOR: Role in cancer, metastasis and drug resistance. Semin Cancer Biol 2019;59:92-111.

24. Jaworski J, Sheng M. The growing role of mTOR in neuronal development and plasticity. Mol Neurobiol 2006;34:205-19.

25. Hoeffer CA, Klann E. mTOR signaling: at the crossroads of plasticity, memory and disease. Trends Neurosci 2010;33:67-75.

26. Wang YY, Yan Q, Huang ZT, et al. Ameliorating Ribosylation-Induced Amyloid- $\beta$ Pathology by Berberine via Inhibiting mTOR/p70S6K Signaling. J Alzheimers Dis 2021;79:833-44.

27. Zhu L, Yuan Q, Zeng Z, et al. Rifampicin Suppresses Amyloid- $\beta$ Accumulation Through Enhancing Autophagy in the Hippocampus of a Lipopolysaccharide-Induced Mouse Model of Cognitive Decline. J Alzheimers Dis 2021;79:1171-84.

28. Ding Y, Liu H, Cen M, et al. Rapamycin Ameliorates Cognitive Impairments and Alzheimer's Disease-Like
Pathology with Restoring Mitochondrial Abnormality in the Hippocampus of Streptozotocin-Induced Diabetic Mice. Neurochem Res 2021;46:265-75.

29. Bi D, Yao L, Lin Z, et al. Unsaturated mannuronate oligosaccharide ameliorates $\beta$-amyloid pathology through autophagy in Alzheimer's disease cell models. Carbohydr Polym 2021;251:117124.

30. Wang D, Cai J, Zeng Z, et al. The interactions between mTOR and NF-кB: A novel mechanism mediating mechanical stretch-stimulated osteoblast differentiation. J Cell Physiol 2020. [Epub ahead of print]. doi: 10.1002/ jcp.30184.

31. Hanagata N, Takemura T, Kamimura K, et al. Effect of immunosuppressants on a mouse model of osteogenesis imperfecta type $\mathrm{V}$ harboring a heterozygous Ifitm 5 c.14C > T mutation. Sci Rep 2020;10:21197.

32. Huang B, Wang Y, Wang W, et al. mTORC1 Prevents Preosteoblast Differentiation through the Notch Signaling Pathway. PLoS Genet 2015;11:e1005426.

33. Li S, Liu B, Zhang L, et al. Amyloid beta peptide is elevated in osteoporotic bone tissues and enhances osteoclast function. Bone 2014;61:164-75.

34. Bhatia V, Sharma S. Role of mitochondrial dysfunction, oxidative stress and autophagy in progression of Alzheimer's disease. J Neurol Sci 2021;421:117253.

35. Weng FL, He L. Disrupted ubiquitin proteasome system underlying tau accumulation in Alzheimer's disease. Neurobiol Aging 202 1;99:79-85.

36. Giovedì S, Ravanelli MM, Parisi B, et al. Dysfunctional Autophagy and Endolysosomal System in Neurodegenerative Diseases: Relevance and Therapeutic Options. Front Cell Neurosci 2020;14:602116.

37. Tran M, Reddy PH. Defective Autophagy and Mitophagy in Aging and Alzheimer's Disease. Front Neurosci 2020;14:612757.

38. Cheng L, Zhu Y, Ke D, et al. Oestrogen-activated autophagy has a negative effect on the anti-osteoclastogenic function of oestrogen. Cell Prolif 2020;53:e12789.

39. di Giacomo V, Cataldi A, Sancilio S. Biological Factors, Metals, and Biomaterials Regulating Osteogenesis through Autophagy. Int J Mol Sci 2020;21:2789.

40. Zheng H, Feng H, Zhang W, et al. Targeting autophagy by natural product Ursolic acid for prevention and treatment of osteoporosis. Toxicol Appl Pharmacol 2020;409:115271.

41. Al-Bari MAA, Xu P. Molecular regulation of autophagy machinery by mTOR-dependent and -independent pathways. Ann N Y Acad Sci 2020;1467:3-20.

42. Li X, Xie X, Yu Z, et al. Bone marrow mesenchymal stem 


\section{Page 16 of 16}

cells-derived conditioned medium protects cardiomyocytes from hypoxia/reoxygenation-induced injury through Notch2/mTOR/autophagy signaling. J Cell Physiol 2019;234:18906-16.

43. Chen S, Jia L, Zhang S, et al. DEPTOR regulates osteogenic differentiation via inhibiting MEG3mediated activation of BMP4 signaling and is involved in osteoporosis. Stem Cell Res Ther 2018;9:185.

44. Chen J, Long F. mTOR signaling in skeletal development and disease. Bone Res 2018;6:1.

45. Martin SK, Fitter S, Dutta AK, et al. Brief report:

Cite this article as: Lin Y, Chen T, Chen J, Fang Y, Zeng C. Endogenous A $\beta$ induces osteoporosis through an mTOR-dependent inhibition of autophagy in bone marrow mesenchymal stem cells (BMSCs). Ann Transl Med 2021;9(24):1794. doi: 10.21037/atm-21-6427

\section{Lin et al. A $\beta$ induces osteoporosis of autophagy in BMSCs}

the differential roles of mTORC1 and mTORC2 in mesenchymal stem cell differentiation. Stem Cells 2015;33:1359-65.

46. Fang D, Wang Y, Zhang Z, et al. Increased neuronal PreP activity reduces $\mathrm{A} \beta$ accumulation, attenuates neuroinflammation and improves mitochondrial and synaptic function in Alzheimer disease's mouse model. Hum Mol Genet 2015;24:5198-210.

(English Language Editor: A. Muijlwijk) 